\title{
Cervical spine clearance after trauma in children
}

\author{
Richard C. E. Anderson, M.D., Peter Kan, M.D., Kris W. Hansen, R.N., \\ AND DOUglas L. BROCKMEYER, M.D. \\ Department of Neurosurgery, Children's Hospital of New York, Columbia University College of \\ Physicians and Surgeons, New York, New York; and Departments of Neurosurgery and Surgery, \\ Primary Children's Medical Center, University of Utah, Salt Lake City, Utah
}

\begin{abstract}
Object. Currently, no diagnostic or procedural standards exist for clearing the cervical spine in children after trauma. The purpose of this study was to determine if reeducation of nonneurosurgical personnel and initiation of a new protocol based on the National Emergency X-Radiography Utilization Study criteria could safely increase the number of pediatric cervical spines cleared of suspected injury without a neurosurgical consultation.

Methods. Data regarding cervical spine clearance in children (ages 0-18 years) after trauma protocol activation at Primary Children's Medical Center between 2001 and 2005 were collected and reviewed. Radiographic and clinical methods of clearing the cervical spine as well as the type and management of injuries were determined for two time frames: Period I (January 2001-December 2003) and Period II (January 2004-July 2005).

Between 2001 and 2003, 95\% of 936 cervical spines were cleared of suspected injury by the neurosurgical service. Twenty-one ligamentous injuries $(2.2 \%)$ and 12 fracture-dislocations $(1.3 \%)$ were detected, with five patients requiring surgical stabilization (0.5\%). Between January 2004 and July 2005, 507 (68\%) of 746 cervical spines were cleared by nonneurosurgical personnel. Six ligamentous injuries $(0.8 \%)$ and 10 fracture-dislocations $(1.3 \%)$ were identified, with three patients $(0.4 \%)$ requiring surgical stabilization. No late injuries were detected in either period.

Conclusions. The protocol used has been effective in enabling detection of cervical spine injuries in children after trauma, with the new protocol increasing by more than $60 \%$ the number of cervical spines cleared by nonneurosurgical personnel. Reeducation with establishment of the new protocols can safely facilitate clearance of the cervical spine by nonneurosurgical personnel after trauma.
\end{abstract}

\section{KEY WORDS • cervical spine injury $\bullet$ spinal cord injury • trauma $\bullet$ fracture • ligament $•$ children}

$\mathrm{C}$ URRENTLY, no national guidelines exist for clearance of the cervical spine in children after trauma. Even after a comprehensive scientific review was conducted and published in Neurosurgery ("Management of pediatric cervical spine and spinal cord injuries" guidelines) in 2002, ${ }^{11}$ insufficient evidence was found to support a standardized diagnostic protocol. ${ }^{6}$

Traditionally, clearance of the cervical spine for suspected injury after trauma has been performed by practitioners in multiple disciplines, including emergency department physicians, orthopedic surgeons, trauma surgeons, and neurosurgeons. At many centers, however, no protocol exists to standardize this process. This often leads to confusion regarding which consulting services are required and creates unnecessary delays in clinical decision making. ${ }^{4}$ In addition, the lack of a standardized protocol can lead to significant variations in procedural methodology that may result in an increased number of missed injuries.

To standardize cervical spine clearance in children af-

Abbreviations used in this paper: $\mathrm{CT}=$ computerized tomography; MR = magnetic resonance; NEXUS = National Emergency $\mathrm{X}$-Radiography Utilization Study. ter trauma, a multidisciplinary protocol was developed and implemented at Primary Children's Medical Center in January 2004. The purpose of this report was to outline the new protocol and describe the methodology, imaging studies, injury types, and personnel involved in pediatric cervical spine clearance. We subsequently compared these data for the periods before (Period I: January 2001-December 2003) and after (Period II: January 2004-July 2005) the protocol was established.

\section{Clinical Material and Methods}

Primary Children's Medical Center is the only Level I pediatric trauma center serving its multistate region. In January 2001, a trauma database was established to collect information about all children between the ages of 0 and 18 years who required evaluation after trauma protocol activation. Part of the data collection includes information about the methodology used in cervical spine clearance.

The following data were collected prospectively: number of children evaluated, children younger than 3 years of age, CT scans performed, MR imaging studies performed, ligamentous injuries, fractures, dislocations, surgical stabil- 


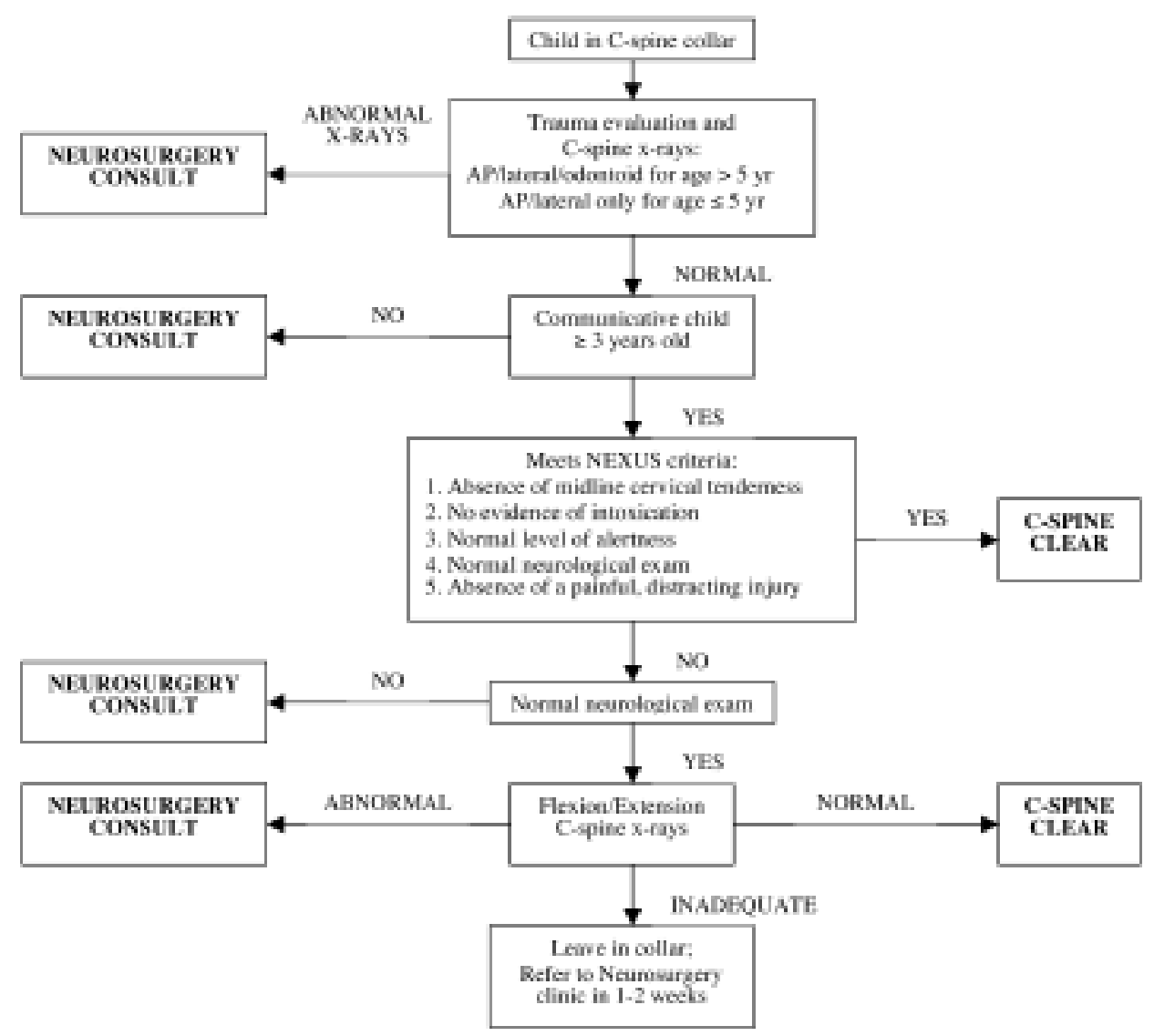

FIG. 1. Flow diagram outlining the current protocol at Primary Children's Medical Center for clearance of the cervical spine of suspected injury after trauma protocol activation in children. AP = anteroposterior.

izations, and injuries detected in a delayed fashion. We also documented which service cleared the cervical spine of suspected injury (neurosurgery, trauma service personnel), or whether the spine could not be cleared during the hospital stay (patient sent home in a cervical collar, with a follow-up visit scheduled in the neurosurgery clinic).

In January 2004, a new protocol was implemented for clearance of suspected injuries in the cervical spine in children. The data collected were then used to compare the methodology of cervical spine clearance before implementation of the protocol (Period I) and after the protocol was initiated (Period II).

\section{Results}

Development of a new protocol for pediatric cervical spine clearance based on the NEXUS criteria ${ }^{12}$ started in August 2003. Approximately 4 months of review and revision were required before approval was given by a multidisciplinary team that included members from general surgery, emergency medicine, and neurosurgery. The protocol was implemented in January 2004 (Fig. 1). Data comparing Periods I and II are summarized in Table 1.

During Period I, 936 children were evaluated after the trauma protocol was activated. Of these, 293 (31\%) were younger than 3 years of age. Standard x-ray films were obtained in all patients, and CT scans were subsequently ob- tained in 81 patients $(9 \%)$ and MR images in $26(3 \%)$. A total of 33 injuries (3.5\%) were found: 21 ligamentous injuries $(2.2 \%)$, eight fractures $(1 \%)$, and four dislocations $(0.5 \%)$. Surgical stabilization was required in five patients $(0.5 \%)$. During this period, 890 cervical spines $(95 \%)$ were cleared by the neurosurgical service, with the remainder cleared by the emergency department or trauma service staff. No late injuries were detected.

During Period II, 746 children were evaluated after activation of the trauma protocol. Of these, $189(25 \%)$ were younger than 3 years of age. Admission CT scans were obtained in 159 (21\%) and MR imaging studies in 34 patients $(4.6 \%)$. Sixteen injuries were detected: six ligamentous injuries $(0.8 \%)$, seven fractures $(0.9 \%)$, and three dislocations $(0.4 \%)$. Three children $(0.4 \%)$ required surgical stabilization. During Period II, 507 cervical spines (68\%) were cleared by nonneurosurgical personnel. In contrast, the cervical spines in 239 children $(32 \%)$ were cleared by neurosurgical personnel during the initial hospitalization, and $66(9 \%)$ were sent home after immobilization in a rigid cervical collar, with a follow-up visit scheduled in the neurosurgery clinic. No late injuries were detected.

\section{Discussion}

In this report we have demonstrated that reeducation and use of our protocol after trauma can safely facilitate clear- 
ance of the cervical spine by nonneurosurgical personnel. The use of this protocol reduced the need for neurosurgical consultation by more than $60 \%$. Similar injury rates were seen during both periods, and no late injuries were detected in either group.

Although many studies describing different methods of clearing the cervical spine in children have been previously published, ${ }^{5,8,9,11,12}$ no current standard of care exists. ${ }^{6}$ Clearing the cervical spine of suspected injury is especially difficult in children because cervical spine injuries are uncommon in this age group, children are often noncommunicative, and there are many normal variations in their anatomy, including pseudosubluxation, absence of lordosis, epiphysial variations, unfused synchondroses, and incomplete ossification. ${ }^{2,3,10}$

Establishment of protocols to clear the pediatric cervical spine of suspected injury has been shown to decrease the time required to accomplish clearance ${ }^{4}$ and reduce the number of missed injuries. ${ }^{7}$ One of the main purposes of our protocol was to define clearly under which circumstances neurosurgical involvement was required. At many centers, neurosurgery is heavily relied on to clear the cervical spine. At a Level I trauma center, this can add up to several "cervical spine clearance" consultations each day, especially during the busy summer months. This places an undue burden on the neurosurgical service and often results in significant delays in diagnosis.

Our data demonstrate that the vast majority of children who are evaluated for cervical spine clearance do not have an injury (positive findings are noted in only $2.9 \%$ of evaluations). In addition, implementation of the protocol at Primary Children's Medical Center reduced the number of neurosurgery consultations for cervical spine clearance by more than $60 \%$. Although we do not have data documenting how long it took to clear the cervical spine of suspected injury, our anecdotal experience strongly supports a reduction in the time required for this procedure after initiation of the new protocol.

Importantly, there were no missed injuries during Period II, confirming the safety of the protocol for use by nonneurosurgical personnel. Although we did not specifically track the number of children requiring flexion-extension radiographs, the majority of children were cleared of suspected injury based on clinical findings. Dynamic radiographs were only obtained in children with a normal mental status and normal results on neurological examination. No complications were seen as a result of performing dynamic imaging studies.

Aside from the reduction in neurosurgical consultations, the only other significant difference seen when comparing the periods before and after implementation of the new protocol is the increase in the number of CT scans ordered by practitioners using the protocol. Of the $159 \mathrm{CT}$ scans performed during Period II, only 73 were obtained at Primary Children's Medical Center. The remaining 86 were performed at other institutions prior to referral. The use of the protocol, therefore, did not result in significantly increased use of CT scanning at Primary Children's Medical Center (9.8\% of all evaluations during Period II compared with 9\% during Period I).

Our protocol offers several advantages. First, it is a standardized protocol that can be followed by personnel from a variety of disciplines in the emergency department or inten-
TABLE 1

Cervical spine clearance in children with trauma based on different protocols*

\begin{tabular}{|c|c|c|}
\hline \multirow[b]{2}{*}{ Factor } & \multicolumn{2}{|c|}{$\begin{array}{c}\text { Period \& } \\
\text { No. of Patients }(\%) \dagger\end{array}$} \\
\hline & I & II \\
\hline trauma protocol activations & 936 & 746 \\
\hline children $<3$ yrs old & $293(31)$ & $189(25)$ \\
\hline CT performed & $81(9)$ & $159(21)$ \\
\hline MRI performed & $26(3)$ & $34(4.6)$ \\
\hline ligamentous injury only & $21(2)$ & $6(0.8)$ \\
\hline fracture & $8(1)$ & $7(0.9)$ \\
\hline dislocation & $4(0.5)$ & $3(0.4)$ \\
\hline surgical stabilization & $5(0.5)$ & $3(0.4)$ \\
\hline late injuries found & 0 & 0 \\
\hline $\begin{array}{l}\text { cervical spine cleared by } \\
\text { nonneurosurgical personnel }\end{array}$ & $46(5)$ & $507(68)$ \\
\hline neurosurgery & $890(95)$ & $239(32)$ \\
\hline sent home in collar; FU in neurosurgery clinic & 0 & $66(9)$ \\
\hline
\end{tabular}

sive care unit setting, thereby simplifying the methodology for cervical spine clearance. Second, it offers a rapid algorithm for cervical spine clearance because the primary service personnel can complete it without subspecialty consultation in most circumstances (68\% of evaluations). Third, the protocol appears safe; no missed injuries were later detected after almost 750 evaluations. Fourth, it clearly defines when neurosurgical involvement is required, thereby significantly reducing the burden on neurosurgical staff.

There are some limitations of our protocol, which are outlined here. In our protocol, all children must undergo plain radiography. The NEXUS report clearly demonstrates that approximately $20 \%$ of children fall into a low-risk category and do not require radiographs..$^{12}$ Nevertheless, because part of the initial trauma evaluation for children as outlined by the American College of Surgeons trauma guidelines includes plain radiographs of the cervical spine, ${ }^{1}$ the films are routinely ordered after activation of the trauma protocol at our institution. Although early versions of our protocol incorporated the NEXUS criteria before radiographic assessment, we were unable to get consensus within our multidisciplinary team for any method that did not incorporate initial radiographic studies after activation of the trauma protocol.

Another limitation is that our protocol does not explain the methodology used by the neurosurgical staff to clear the cervical spine of suspected injury. The purpose of our protocol was not to outline specific methods that can be used to clear the cervical spine in children with abnormal results on x-ray films, those with neurological injuries, or those who are noncommunicative (that is, children $<3$ years old or in a coma). Rather, our goal was to facilitate the process of cervical spine clearance in children by developing a standardized protocol that limits the number who require subspecialty consultation to those with the greatest chance of an injury.

\section{Conclusions}

Currently, no national guidelines exist for clearance of 
the cervical spine in children after trauma. In this report, we have demonstrated that education and establishment of a standardized protocol can safely facilitate clearance of the cervical spine by nonneurosurgical personnel after trauma. The use of this protocol reduced the need for neurosurgical consultation by more than $60 \%$. Similar injury rates were seen during both periods, and no late injuries were detected in either group.

\section{References}

1. Alexander RH, Proctor HJ, American College of Surgeons Committee on Trauma: Advanced trauma life support program for physicians: ATLS. Chicago: American College of Surgeons, 1993

2. Cattell HS, Filtzer DL: Pseudosubluxation and other normal variations in the cervical spine in children. A study of one hundred and sixty children. J Bone Joint Surg Am 47:1295-1309, 1965

3. Fesmire FM, Luten RC: Evaluation of the pediatric cervical spine, in Harwood-Nuss AL (ed): The Clinical Practice of Emergency Medicine, ed 2. Philadelphia: Lippincott-Raven, 1996, pp 1197-1201

4. Frank JB, Lim CK, Flynn JM, et al: The efficacy of magnetic resonance imaging in pediatric cervical spine clearance. Spine 27: 1176-1179, 2002

5. Jaffe DM, Binns H, Radkowski MA, et al: Developing a clinical algorithm for early management of cervical spine injury in child trauma victims. Ann Emerg Med 16:270-276, 1987

6. Joint Section on Disorders of the Spine and Peripheral Nerves of the AANS/CNS: Management of pediatric cervical spine and spinal cord injuries. Neurosurgery 50:S85-S99, 2002

7. Lee SL, Sena M, Greenholz SK, et al: A multidisciplinary approach to the development of a cervical spine clearance protocol: process, rationale, and initial results. J Pediatr Surg 38: 358-362, 2003

8. McCarthy C, Oakley E: Management of suspected cervical spine injuries-the paediatric perspective. Accid Emerg Nurs 10: 163-169, 2002

9. Ross SE, Schwab CW, David ET, et al: Clearing the cervical spine: initial radiologic evaluation. J Trauma 27:1055-1060, 1987

10. Shaw M, Burnett $H$, Wilson A, et al: Pseudosubluxation of $\mathrm{C} 2$ on C3 in polytraumatized children-prevalence and significance. Clin Radiol 54:377-380, 1999

11. Slack SE, Clancy MJ: Clearing the cervical spine of paediatric trauma patients. Emerg Med J 21:189-193, 2004

12. Viccellio P, Simon H, Pressman BD, et al: A prospective multicenter study of cervical spine injury in children. Pediatrics 108: E20, 2001

Manuscript received December 15, 2005.

Accepted in final form January 17, 2006.

Address reprint requests to: Richard C. E. Anderson, M.D., The Neurological Institute, Room 213, 710 West 168th Street, New York, New York 10032. email: rca24@ columbia.edu. 